\title{
Correspondence
}

\section{Intubation using the ProSeal laryngeal mask airway and a Cook airway exchange catheter set}

To the Editor:

The newest laryngeal mask airway (LMA), the ProSeal LMA (PSLMA, The Laryngeal Mask Company, San Diego CA, USA) was not designed as an intubation device but to increase the periglottic seal and to provide access to the gastrointestinal tract. ${ }^{1-3}$ Nevertheless, the anesthesiologist may be confronted with the situation of having to intubate the trachea with the PSLMA in place. We report a method to change the PSLMA to an endotracheal tube.

Our initial experience in using the PSLMA in ten consecutive patients demonstrated an altered glottic view. Using a 3.4-mm OD pediatric fibreoptic bronchoscope (FOS, Pentax, Tokyo, Japan), we examined the glottic view with a \#5 PSLMA installed. Using the scoring system described by Dr. Brain, et al., ${ }^{1}$ three patients had grade 1 view, four had a grade 2 view, and three had a grade 3 view. The PSLMA may induce anterior laryngeal structure movement and rotation of the arytenoids relative to the cricoid cartilage, potentially explaining the altered view. ${ }^{4}$

We used a Cook airway exchanger kit, Arndt set, to overcome these difficulties to exchange the \#5 PSLMA for an endotracheal tube. We were requested to change a \#5 PSLMA to an $8.0-\mathrm{mm}$ ID endotracheal tube (ETT) for postoperative ventilation in a 55 -yr-old, 82 $\mathrm{kg}$ male undergoing a right hemicolectomy. A \#2 view was seen. The Cook airway exchange kit contains a 144-cm guide wire, a \#11 French 70-cm exchange stylet, rapifit adapters and a bronchoscope adapter. Following bronchoscopy, the guide wire was advanced easily through the FOS suction port into the trachea under direct visualization. The FOS was removed and the exchange stylet advanced over the wire. The PSLMA was removed and an $8.0-\mathrm{mm}$ ID ETT advanced over the stylet. The stylet was removed when carbon dioxide was detected and the lungs ausculted.

The role of the PSLMA in the difficult airway is yet to be defined. The double cuffed aperture bar free PSLMA has specific structural differences from the standard LMA that may increase the difficulty of using it as a conduit for intubation.
Adrian Matioc MD

George A. Arndt MD

Madison, WI

\section{References}

1 Brain AIJ, Verghese C, Strube PJ. The LMA 'ProSeal'a laryngeal mask with anesophageal vent. Br J Anaesth 2000; 84: 650-4.

2 Brimacombe JR, Keller $C$. The ProSeal laryngeal mask airway: a randomized, crossover study with the standard laryngeal mask airway in paralyzed, anesthetized patients. Anesthesiology 2000; 93: 104-9.

3 Keller C, Brimacombe J. Mucosal pressure and oropharyngeal leak pressure with the ProSeal versus laryngeal mask airway in anaesthetized paralyzed patients. $\mathrm{Br} \mathrm{J}$ Anaesth 2000; 85: 262-6.

4 Nandi PR, Nunn JF, Charlesworth CH, Taylor SJ. Radiological study of the laryngeal mask. Eur J Anaesth 1991; Suppl. 4: 33-9.

5 Walburn MB, Cornes J, Ryder IG. Fibreoptic intubation through a laryngeal mask airway facilitated by a guide wire. Anesthesia 2000; 55: 1027-8.

\section{Monitoring of retrohepatic inferior vena caval pressure predicted postopera- tive hematoma after hepatic surgery}

\section{To the Editor:}

We describe a case of postoperative hematoma diagnosed by monitoring of the retrohepatic inferior vena caval (IVC) pressure in the intensive care unit (ICU). Such monitoring may be useful for the perioperative management of patients undergoing hepatic surgery.

After undergoing hepatic resection for hepatocellular carcinoma, a 63-yr-old, $163 \mathrm{~cm}, 70 \mathrm{~kg}$ male became hypotensive in the ICU. At that time, we noticed an increased retrohepatic IVC pressure $(15 \mathrm{mmHg})$ and an increased pressure difference with the superior IVC pressure $(1 \mathrm{mmHg})$. Besides, hypovolemia was present as evidenced by pulmonary artery pressure monitoring. Therefore, we speculated a postoperative intra-abdominal hematoma was obstructing the IVC, cephalad from the catheter tip for retrohepatic IVC pressure monitoring. Computed tomographic scanning followed by inferior vena cavography (Figure) revealed the mass, resulting in a successful reoperation for hematoma removal. 